\title{
Hacia una evaluación de la reflexión pedagógica desde la escritura académica
}

Towards an Evaluation of Pedagogical Reflection from Academic Writing

Artículo de investigación | Research Article

Fecha de recepción: 04 de junio de 2020

Fecha de aceptación: 18 de febrero de 2021

Fecha de disponibilidad en línea: julio de 2021

doi: 10.11144/Javeriana.m14.herp

Nicole SChNitZler-SOMmerfeld nicole.schnitzler@mayor.cl

Escuela de Educación, Facultad de Humanidades, Universidad Mayor, Chile (D) ORCID: https://orcid.org/0000-0002-3339-1146

Paulina Núñez-Lagos

paulina.nunez@umayor.cl

Escuela de Educación, Facultad de Humanidades, Universidad Mayor, Chile

(iD) ORCID: https://orcid.org/0000-0002-6991-6768

Para citar este artículo | To cite this article

Schnitzler-Sommerfeld, N. \& Núñez-Lagos, P. (2021). Hacia una evaluación de la reflexión pedagógica desde la escritura académica. magis, Revista Internacional de Investigación en Educación, 14, 1-25. doi: 10.11144/Javeriana.m14.herp 


\title{
Resumen
}

Este artículo de investigación describe el diseño y aplicación de un instrumento de evaluación del pensamiento crítico desde la escritura académica como método para desarrollar la reflexión pedagógica. A partir de una metodología cuantitativa, con alcance descriptivo, se analizaron 265 ensayos de estudiantes chilenos de pedagogía. La capacidad de relacionar de forma consistente y coherente los argumentos, y la habilidad para articular el punto de vista propio con otras perspectivas sistemáticamente, resultaron los mayores predictores en la elaboración de un texto escrito con un alto nivel de pensamiento crítico (Martin \& White, 2003; Snow \& Uccelli, 2009; Youngs \& Bird, 2010).

\section{Palabras clave}

Pensamiento crítico; educación superior; escritura; formación de docentes; ciencias de la educación

\begin{abstract}
This research article describes the design and application of an instrument for evaluating critical thinking from academic writing as a method to develop pedagogical reflection. This research was based on a quantitative methodology, with a descriptive scope, where 265 essays, written by Chilean pedagogy students, were analyzed. The ability to consistently and coherently relate arguments, and the ability to systematically articulate one's point of view with other perspectives, were the greatest predictors in the development of a written text with a high level of critical thinking (Martin \& White, 2003; Snow \& Uccelli, 2009; Youngs \& Bird, 2010).
\end{abstract}

\section{Keywords}

Critical thinking; higher education; writing; teacher education; educational sciences 
Descripción del artículo | Article description

Artículo de investigación derivado del proyecto Desarrollo

de la reflexión pedagógica desde la escritura académica.

\section{Introducción}

Las habilidades y actitudes para el siglo XXI exigen que las instituciones de educación superior, y en específico los programas de formación docente, habiliten a los futuros profesionales para que a partir de una mirada holística desarrollen soluciones rápidas y coherentes a los desafíos del aula, tales como la heterogeneidad cultural e idiomática de los estudiantes, los distintos tipos y ritmos de aprendizaje de los mismos, y la interrelación con el ámbito familiar, entre otros.

En términos teóricos, generar estrategias para desenvolverse en las diversas situaciones que conlleva la práctica docente, y que estas se fundamenten en marcos interpretativos y críticos que consideren tanto la diversidad dentro del aula como las representaciones institucionales y gubernamentales del quehacer pedagógico (Salinas et al., 2018), significa aplicar un sistema educativo basado en la reflexión pedagógica, entendida esta como la verbalización, oral o escrita, de una propuesta de acción, producto de un proceso en el que se negocia, con audiencias concretas o potenciales, una solución a un problema o desafío del ámbito pedagógico (Lara, 2019; Salinas et al., 2018; Schön, 1992).

A pesar de que, desde la perspectiva pedagógica contemporánea, la reflexión pedagógica es un aspecto que se evalúa formalmente en la práctica y en la formación docente', tanto en Chile como en otras partes del mundo las capacidades de reflexión pedagógica resultan preocupantes, sobre todo en los profesores novatos (Concha et al., 2013; DarlingHammond \& Bransford, 2005) y en los estudiantes que integran las carreras de pedagogía. Esta situación se refleja en los resultados de las distintas

1 Con el objetivo de implementar un sistema educativo basado en la reflexión pedagógica, esta se mide explícitamente en los profesores que ejercen dentro de Chile, en el marco de la Ley 20.903 (Sistema de Evaluación del Desempeño Profesional Docente) y, además, de manera obligatoria, en los estudiantes que egresan de carreras de pedagogía a través de la Evaluación Nacional Diagnóstica de la Formación Inicial Docente (END-FID). Ambas evaluaciones son aplicadas por el Centro de Perfeccionamiento, Experimentación e Investigaciones Pedagógicas (CPEIP) del Ministerio de Educación de Chile (MINEDUC). 
tareas académicas que implican reflexión pedagógica dentro de las instituciones universitarias, y en las evaluaciones a nivel nacional, tales como la Evaluación Nacional Diagnóstica de la Formación Inicial Docente (END-FID), una prueba que constituye un requisito obligatorio para que los futuros profesores obtengan su título profesional ${ }^{2}$. Estos resultados poco satisfactorios (END-FID, 2016-2018) han derivado en la creación de diversos programas de alfabetización pedagógica elaborados tanto de manera local, por cada establecimiento formador de profesores, así como de manera transversal por el Ministerio de Educación de Chile.

No obstante, a pesar de las acciones y la preocupación en torno a la reflexión pedagógica por parte de los formadores de profesores y las instituciones que los respaldan, existen escasos estudios que reporten instrumentos válidos para evaluar la reflexión pedagógica (Concha et al., 2013; Youngs \& Bird, 2010), y en la literatura no se encuentran suficientes sistematizaciones analíticas respecto del desarrollo progresivo de las habilidades implicadas en dicha reflexión durante las distintas etapas de la formación docente (Ossa-Cornejo et al., 2017), por lo que es posible afirmar que permanecen ciertas incertidumbres y obstáculos para evaluar y realizar reflexión pedagógica en términos concretos.

Ante tal problema, este estudio surge como una propuesta para evaluar las competencias escritas de los estudiantes recién ingresados a la carrera de pedagogía e identificar los niveles de desarrollo de pensamiento crítico, como base de la reflexión pedagógica; con el propósito de establecer un punto de partida a partir del cual sea posible realizar un posterior seguimiento. El objetivo general de este análisis es describir el diseño y la aplicación de un instrumento de evaluación de pensamiento crítico a través de la escritura, del cual se desprenden los siguientes objetivos específicos:

a. Diseñar un instrumento que permita examinar de manera cuantitativa el rendimiento en las habilidades de pensamiento crítico que sustentan la reflexión pedagógica.

b. Describir el desempeño de los estudiantes que recién están comenzando su formación docente en las habilidades de pensamiento crítico que les permitirán desarrollar una futura reflexión pedagógica.

2 La Ley de Sistema de Desarrollo Docente (20.903) establece que los estudiantes de pedagogía deben rendir dos evaluaciones diagnósticas: la primera evalúa competencias de redacción y es aplicada por la universidad al inicio de la carrera, y la segunda (END-FID) es ejecutada por el CPEIP los doce meses que anteceden al año del egreso, con el objetivo de entregar información a las instituciones para elaborar planes de mejora de sus programas formativos (MINEDUC, 2019). 
c. Determinar la incidencia de cada habilidad o dimensión del instrumento en un producto escrito que evidencie un alto nivel de pensamiento crítico.

d. Estimar las relaciones entre las dimensiones textuales (coherencia, adecuación léxica y ortografía) y un producto escrito que evidencie un alto nivel de pensamiento crítico.

El interés respecto al modo en que la escritura se relaciona directamente con el proceso de reflexión pedagógica sirve, entre varios aportes metodológicos, para evaluar su desarrollo en un programa de formación docente (Luk, 2008; Snow \& Uccelli, 2009; Youngs \& Bird, 2010) y particularmente este estudio, cuantitativo con alcance descriptivo, integra las nociones de escritura, argumentación razonada y pensamiento crítico para sistematizar las habilidades y los procesos que se ponen en práctica al enfrentar una tarea que implica reflexión pedagógica. Esta asociación conceptual se sustenta en la idea de que la complejidad intrínseca del pensamiento crítico radica en su carácter dialógico, manifestándose principalmente a través del recurso de la contraargumentación, y siendo más complejo en la modalidad escrita dado el carácter abstracto de los participantes que representan diferentes posturas, pues implica la construcción de un texto heteroglósico que dé cuenta de estas voces o posiciones de audiencias tangibles y/o hipotéticas (García \& Barrientos, 2009; Martin \& White, 2003).

Por otro lado, y con el propósito de vincular esta investigación con las herramientas y los recursos que existen en Chile actualmente para desarrollar y evaluar ejercicios de reflexión pedagógica, el estudio considera significativa la END-FID, no solo por los resultados que levanta respecto al desempeño de los estudiantes en torno a esta, sino, porque, además constituye un parámetro respecto de lo que los estudiantes deben lograr en dicho tema ${ }^{3}$. De todas maneras, cabe recalcar que la necesidad de generar

3 Específicamente, la END-FID consta de tres partes: la Prueba de Conocimientos Pedagógicos Generales (PCPG), la Prueba de Conocimientos Disciplinarios y Didácticos (PCDD) y un cuestionario complementario de percepción. Dentro de la primera prueba mencionada existe una pregunta de reflexión pedagógica, cuyo formato es de respuesta abierta, en la que el o la estudiante debe resolver un caso enunciado, como, por ejemplo, una situación en la que un profesor jefe de educación media debe organizar las fiestas patrias chilenas, enfrentándose a un curso compuesto por estudiantes de distintas nacionalidades y culturas. Los estudiantes de cuarto año de pedagogía deben describir las estrategias, actividades y recursos pedagógicos que utilizarían para resolver la situación, en el caso ejemplificado para favorecer la inclusión de sus estudiantes, fundamentando sus decisiones. 
instrumentos estandarizados para desarrollar y evaluar la reflexión pedagógica no tiene únicamente un fin académico y de medición, sino que se proyecta en la formación de profesores capaces de interactuar críticamente con todos los actores y en todos los contextos del mundo pedagógico laboral, de forma que puedan desenvolverse a partir de los requerimientos e ideales de la pedagogía contemporánea.

\section{Marco de referencia}

\section{Reflexión pedagógica y pensamiento crítico}

La reflexión pedagógica, entendida como la puesta en práctica del pensamiento crítico en el ámbito pedagógico laboral, ha tenido una gran relevancia durante la última década en los perfiles de egreso de las carreras de pedagogía (Beauchamp, 2015; Collin et al., 2013). Esta competencia integra tanto recursos emocionales como habilidades cognitivas y metacognitivas requeridas para enfrentar una experiencia educacional desafiante, que alcanza su fin cuando se resignifica el objeto de reflexión (Lara, 2019; Salinas et al., 2018; Schön, 1992). En tanto proceso, la reflexión pedagógica constituye un diálogo co-constructivo, en el que interactúan las distintas posiciones, voces o actores que exponen sus puntos de vista frente a una situación problemática del área pedagógica.

Al aterrizar este concepto a una tarea de escritura, es relevante considerar ciertos presupuestos acerca del lenguaje, tales como la construcción social del significado y la naturaleza intertextual del discurso, pues dado el rasgo dialógico que por esencia define a la reflexión pedagógica, interesa el modo en que en los textos se negocian significados con las audiencias (Bajtín, 1981) y se enuncia un punto de vista fundamentado, más que el contenido ideacional y el valor de verdad que se asocia a él. Como producto, la reflexión pedagógica suele presentarse en forma de una propuesta verbal conducente a una acción para enfrentar una problemática o experiencia desafiante, que puede estar inscrita en diversos géneros discursivos, tales como informes, diarios murales, encuestas, reuniones de áreas, etc., y dentro de la formación docente en géneros discursivos específicos de la misma, tales como estudios de caso, crónica docente y portafolio (Jarpa \& Becerra, 2019; Núñez \& Espejo, 2005; Tapia-Ladino \& Burdiles Fernández, 2009). Estos géneros pedagógicos conducen a objetivos de aprendizaje particulares, en tareas de escritura que se les solicitan a los estudiantes para la adquisición de habilidades cognitivas involucradas en la reflexión pedagógica (Jarpa \& Becerra, 2019).

De esta manera, hay varias consideraciones teóricas desde las que emergen habilidades y procesos específicos involucrados en la reflexión 
pedagógica4. A partir de los trabajos de Pérez Gómez (2007), Larrivee (2008), Rodrigues (2013) y Lamas \& Vargas-D’Uniam (2016), se desprenden varias propuestas: niveles de reflexión de Van Manen (1977), de Hatton \& Smith (1995) y de Schön (1998), tipos de pensamiento reflexivo de SparksLanger et al. (1990), niveles de práctica docente de Larrivee (2008) y niveles de escritura reflexiva de Moon (2007), que si bien difieren en la explicitación de los momentos en los que se produce la acción y la reflexión como tal, permiten sistematizar el proceso en etapas o niveles. La reflexión pedagógica implicaría entonces: (1) el desarrollo de una acción enfocada hacia la resolución de un problema (Freire, 1997; Hatton \& Smith, 1995; Moon, 2007; Schön, 1992), pero distinguiendo la solución de la reflexión en sí, requiriendo un proceso más metacognitivo que permita distanciarse de la acción; (2) identificar el problema; (3) considerar el contexto socio-históricopolítico-cultural; (4) abarcar las diversas perspectivas o actores involucrados en la situación; y, por último, (5) valorar la acción realizada como una manera de resignificar el conflicto para construir una propuesta de resolución.

Las competencias enumeradas constituyen lo que muchos autores (Freire, 1997; Van Manen, 1977; Zeichner, 1987) han acoplado bajo el término de reflexión crítica, refiriéndose a una línea educativa que integra la teoría con el contexto situacional inmediato (Rodrigues, 2013), para desplegar un sistema sensible y reactivo a todos los cambios dentro y fuera del mundo educativo. Es por esta razón que también las mismas se acogen en este trabajo para la construcción de un instrumento que permita evaluar y, posteriormente, enseñar y desarrollar la reflexión pedagógica en los estudiantes de pedagogía, de manera que los y las futuras profesoras sean capaces de entregar respuestas atingentes a la realidad en la que están inscritos.

\section{La evaluación de la reflexión pedagógica desde el discurso escrito}

Dentro de los aspectos más consensuados y respaldados que se manejan en torno a los procesos actuales de evaluación y desarrollo de la reflexión pedagógica, destaca la consideración de la escritura como medio privilegiado para la realización de estos procesos, no solo porque sin duda los textos reflejan las habilidades implicadas en la reflexión pedagógica, sino porque, además, las habilidades discursivas de construcción y organización de un texto se relacionan directamente con el proceso de producción de reflexiones pedagógicas (Concha et al., 2013; Fund, et al., 2002;

4 Tanto a partir de las nociones de reflexión pedagógica como de los parámetros de escritura y argumentación se desprenden trece habilidades que progresivamente se van enumerando en este apartado para fundamentar el diseño del instrumento. 
Luk, 2008; Youngs \& Bird, 2010), y, adicionalmente, en términos prácticos, los textos escritos sirven como evidencia para medir progresivamente y elaborar propuestas didácticas basadas en datos empíricos.

Una de las actividades de escritura existentes que permite desarrollar y evaluar las habilidades involucradas en la reflexión pedagógica es la construcción de textos predominantemente argumentativos, que frecuentemente se traducen en la producción de ensayos ${ }^{5}$ y en una variedad de géneros discursivos del ámbito pedagógico en la formación docente. Cuando los escritos o segmentos textuales cumplen la función de enunciar una reflexión pedagógica, el foco es seleccionar los recursos discursivos y retóricos pertinentes que permiten a los estudiantes cohesionar sus ideas a partir de relaciones lógicas, marcadores discursivos y conectores, entre otros, para integrar sus conocimientos teóricos, experienciales e ideológicos. Este proceso puede sintetizarse en la noción de argumentación razonada que adopta esta investigación (Silvestri, 2001), la cual exige el despliegue de un razonamiento compatible con el desarrollo de una tesis, y, discursivamente, requiere que el razonamiento se enuncie según las convenciones científicas de la academia, la institución universitaria y el área de especialidad (Parodi, 2010; Snow \& Uccelli, 2009).

Desde el punto de vista conceptual, existe un estrecho vínculo entre argumentación y pensamiento crítico, y particularmente esta investigación se desarrolla en torno a la visión de que varias habilidades, que tradicionalmente se han reconocido en los procesos de argumentación, implican o provienen del pensamiento crítico, tales como enunciar con claridad una tesis, evaluar los argumentos e identificar contraargumentos (Mota de Cabrera, en Córdova et al., 2016). Si se considera una relación interdependiente entre el pensamiento crítico y la argumentación, es posible concebir a la escritura epistémica como una acción que exige la activación de todos los procesos y las habilidades que subyacen a la argumentación y al pensamiento crítico, consolidando así el proceso de alfabetización académica ${ }^{6}$. Esta función epistémica se evidencia más aún si, como se

5 Si bien el ensayo argumentativo puede resultar un género muy amplio, no necesariamente propio del ámbito pedagógico, tiene sentido en el contexto académico y sobre todo de ingreso a la pedagogía como diagnóstico, pues apunta a evaluar las competencias escritas que los estudiantes ya han adquirido en la escolaridad, y que, a futuro, les permitirán desarrollar otras habilidades de pensamiento crítico y reflexión pedagógica (Marinkovic, 2007).

6 Ya la definición de pensamiento crítico, dentro del espectro conceptual influenciado por una gran variedad cultural proveniente de diversas tradiciones filosóficas, se basa en la rama de las disciplinas retóricas y en la figura argumentativa (García \& Barrientos, 2009), y gran parte de sus nociones tienen un tinte pedagógico, aun cuando provienen de otras disciplinas. De esta manera, las aproximaciones teóricas respecto al pensamiento crítico no solo se refieren a un 
mencionó anteriormente, el escrito o segmento textual intenta integrar las posturas (heteroglosia), como ocurre particularmente en las tareas de reflexión pedagógica.

Es por esto que en las últimas décadas ha existido un fuerte interés por la escritura, considerada en su conjunto desde una perspectiva sociocomunicativa, siendo relevantes las situaciones de interacción en las que se insertan las tareas, las que han dado como resultado el desarrollo de diversos programas específicos de escritura académica (Camps \& Castello, 2013; Navarro, 2014; Parodi, 2010; Sologuren, 2019; Venegas, Zamora \& Galdames, 2016). Paralelamente, se han estudiado las dificultades, predominantemente argumentativas, que presentan los estudiantes al realizar tareas de escritura, e incluso los mismos alumnos y alumnas que ingresan a la educación superior tienen conciencia de que no han desarrollado la capacidad de elaborar un pensamiento crítico mediante la lectoescritura para el nivel requerido en ese contexto educacional (Navarro, 2014).

Esta investigación se refiere a las habilidades que fundamentan la reflexión pedagógica en términos de pensamiento crítico, pues este cimenta un lenguaje y un discurso propio que crea un espacio simbólico de negociación en un tiempo y espacio determinado, el cual no solo se representa, sino que se desarrolla durante la producción textual, debido a las estrategias y mecanismos que se traman en la formulación del mensaje. Así, como plantean Van Dijk \& Kinstch (1983) desde la lingüística del texto, el pensamiento crítico textual es la representación en paralelo del contenido de los mensajes, la elección léxica para concretizarlo, la forma sintáctica, la ideología y estructuración de diversas perspectivas y visiones de mundo, y la representación de sonidos. Es en la línea argumentativa del pensamiento crítico donde subyace una práctica educativa que promueve el aprendizaje y adquisición de la justificación, el apoyo, el desarrollo de una idea, la valoración, la consideración de diversas alternativas, el convencimiento y la contraargumentación por parte de los estudiantes, además del desarrollo de la lógica formal y todos los procesos argumentativos que esta implica (García \& Barrientos, 2009).

\section{Metodología}

Ante las dificultades para evaluar, cuantificar y, por ende, formar en la reflexión pedagógica en el contexto universitario, esta investigación se

conjunto de procesos cognitivos y percepciones de realidad, sino que especifican estos procesos como competencias académicas relacionadas directamente con la lectura y escritura (Scriven \& Fisher, 1997), que construyen y generan discursos transformadores (Freire, 1997). 
enmarca en un proyecto mayor, que busca generar herramientas progresivas de evaluación y desarrollo de reflexión pedagógica desde un medio escrito. Tales actividades de escritura deben exigir la integración de conocimientos, razonamiento lógico y negociación de significado entre otros aspectos de la argumentación razonada y el pensamiento crítico, para preparar profesores expertos en reflexión pedagógica, y sistematizar las habilidades implicadas en esta, que se van desarrollando en cada etapa de la formación docente. En particular, el presente trabajo se contextualiza en un nivel inicial, como un análisis diagnóstico de las capacidades de los estudiantes que recién ingresan a la carrera de pedagogía.

Con el propósito de responder a los objetivos de investigación mencionados en la introducción, se diseñó un instrumento para evaluar textos escritos en función de las habilidades discursivas del pensamiento crítico que fundamentan la reflexión pedagógica.

\section{Muestra}

La muestra se compone de 265 textos pertenecientes a estudiantes recién ingresados a una universidad de carácter privado, ubicada en la Región Metropolitana de Chile, como alumnos y alumnas de primer año (excluyendo a quienes estudiaron otras carreras previamente), matriculados en seis programas de pedagogía: Pedagogía en Educación Física, Deportes y Recreación Básica y Media ( $n=89 ; 33.6 \%$ ), Pedagogía en Inglés Básica y Media ( $n=48 ; 18.1 \%$ ), Pedagogía en Educación Diferencial ( $n=46$; $17.3 \%$ ), Pedagogía en Artes Musicales ( $n=35 ; 13.2 \%)$, Psicopedagogía $(n=24 ; 9.0 \%)$, Pedagogía en Educación Parvularia $(n=23 ; 8.6 \%)$. Además de excluir de la muestra aquellos estudiantes que no estuviesen recién egresados del colegio, evidentemente también se apartaron los textos ilegibles, principalmente por problemas de caligrafía o dificultades en su visualización.

\section{Instrumento}

Para evaluar específica y cuantitativamente las habilidades que sustentan la reflexión pedagógica, se elaboró una rúbrica que pudiese ser aplicada, en primera instancia, para la revisión de ensayos argumentativos, considerando los parámetros de los Niveles de Desempeño de la END- FID (2017, 2018 y 2019), y dimensiones discursivas que dan cuenta tanto de la lógica argumentativa y el pensamiento crítico del desarrollo textual, como del manejo consistente de las normas de expresión escrita y los recursos de cohesión, para analizar los textos global y holísticamente. Concretamente, de las diversas líneas que confluyen en el apartado del marco teórico, es posible desprender 13 competencias que se sintetizan a continuación: 
(1) desarrollar habilidades para la resolución de problemas; (2) identificar el problema; (3) considerar el contexto socio-histórico-político-cultural; (4) considerar diversas perspectivas o actores involucrados en la situación; (5) valorar la resolución; (6) evaluar los argumentos; (7) identificar los contraargumentos; (8) integrar diversas perspectivas y visiones de mundo; (9) desarrollar y argumentar una idea; (10) realizar juicios y valores; (11) considerar diversas alternativas; (12) argumentar y persuadir; y (13) contraargumentar. Al categorizar estas competencias dentro de un proceso de escritura argumentativo, resultan siete dimensiones como posibles parámetros de evaluación de un texto escrito a partir de las habilidades que sustentan la reflexión pedagógica:

Tabla I

Dimensiones del Instrumento de evaluación de habilidades escritas de pensamiento crítico (I1)

\begin{tabular}{l} 
Dimensión \\
\hline I. Identificación del problema según contexto \\
\hline II. Planteamiento de tesis \\
\hline III. Relación argumentativa \\
\hline IV. Identificación de objeciones \\
\hline V. Valoración \\
\hline VI. Inferencia de resolución \\
\hline VII. Análisis sistémico
\end{tabular}

Fuente: elaboración propia

Cabe mencionar que los aspectos semánticos y formales de escritura, es decir, las normas de expresión escrita y los recursos de coherencia fueron evaluados a partir de otra rúbrica, debido a las facilidades de corrección que esto supuso en el contexto de las distintas líneas de investigación que el proyecto global compone. Este instrumento (en adelante Instrumento de evaluación de aspectos formales y textuales) contempló las dimensiones de: (1) coherencia oracional, (2) coherencia interpárrafos, (3) uso de conectores, (4) convenciones ortográficas, y (5) variedad y adecuación léxica.

Los textos fueron producidos en el contexto de la evaluación diagnóstica de competencias de redacción en el marco de la Ley 20.903, con la intensión de redactar un texto argumentativo de carácter formal, en el que se expresara claramente un punto de vista y se desarrollaran argumentos, a partir de la lectura de un breve texto de opinión. En las instrucciones, se les solicitaba a los estudiantes adoptar una postura, pero se les indicó que esta no sería evaluada como contenido, sino más bien según la capacidad de 
expresar y justificar el punto de vista. Además, durante los sesenta minutos que tuvieron los estudiantes para cumplir con la tarea requerida dispusieron de una rúbrica de evaluación de parámetros argumentativos y de escritura que se explicó antes de iniciar la prueba.

\section{Procedimiento}

Posterior a la instancia de evaluación diagnóstica y al diseño del instrumento para los fines investigativos específicos, se realizó un proceso de validez de contenido del mismo por parte de dos especialistas del área de educación y dos lingüistas, que consistió en la aplicación individual de la rúbrica a tres textos de la muestra y en una valoración cuantitativa a través de una escala de Likert de 5 puntos (siendo 1 ausente o requiere demasiadas modificaciones y 5 presente en su totalidad) para la apreciación de relevancia, pertinencia, claridad, y suficiencia de cada dimensión, así como una valoración cualitativa mediante una sección abierta de comentarios y sugerencias. Esto derivó en ajustes de la propuesta inicial y en un proceso de pilotaje en tres etapas de doble corrección, para afinar la fiabilidad del instrumento: dos etapas de revisión de 25 textos de la muestra por parte de las investigadoras, entre las que se realizaron algunas modificaciones, y otra de doble corrección de 100 textos de la muestra por parte de revisores externos, que también contribuyó para algunos cambios finales.

Una vez concluida la fase de elaboración del instrumento, se procedió a la revisión del 100\% de los textos por parte de correctores expertos, siendo resueltas las inconsistencias por un juez externo, y, posteriormente, al examen cuantitativo de los resultados. Al ser una investigación con un alcance descriptivo-correlacional, se realizó el análisis a partir de la correlación de las dimensiones de cada rúbrica, de manera que los resultados del estudio fueran un indicador del estado de los alumnos y alumnas de primer año, respecto a las habilidades antes mencionadas. En este sentido, para cuantificar los datos se tuvieron en cuenta los rangos de puntuación de cada rúbrica: 0-4 en el Instrumento de evaluación de habilidades escritas de pensamiento crítico (I1), siendo 4 el puntaje máximo por dimensión, y consecuentemente 28 el posible mejor total; y 0-3 en la rúbrica para evaluar los aspectos formales de escritura, donde, en este caso, el puntaje más alto es el valor 3, y 15 el que representa el logro cabal de todas las dimensiones.

El estudio y análisis estadístico de los datos, así como la posterior extracción de resultados se hizo por medio de la utilización de estadísticos descriptivos, y para comprobar la normalidad de la muestra se aplicó la prueba de Kolmogorov-Smirnov (K-S), con $p<0,001$. El análisis de datos se realizó a través del paquete estadístico IBM SPSS V.21. 


\section{Resultados}

En adelante, se presentan los resultados de acuerdo con los objetivos específicos:

\section{a. Diseñar un instrumento que permita examinar de manera cuanti- tativa el rendimiento en las habilidades que sustentan la reflexión pedagógica.}

Tabla 2

Instrumento de habilidades escritas de pensamiento crítico (11)

\begin{tabular}{|c|c|c|}
\hline Dimensión & Ptje & Descripción \\
\hline \multirow[t]{5}{*}{$\begin{array}{l}\text { I. Identificación } \\
\text { del problema } \\
\text { según contexto }\end{array}$} & 4 & $\begin{array}{l}\text { El problema se identifica y desarrolla adecuada, sistemática y explícitamente según } \\
\text { datos relevantes para su resolución y contexto (tópico general, tópico específico y } \\
\text { propósito argumentativo). }\end{array}$ \\
\hline & 3 & $\begin{array}{l}\text { El problema se identifica y desarrolla adecuada y explícitamente, pero presenta una } \\
\text { desviación aislada y no justificada del tema específico. }\end{array}$ \\
\hline & 2 & $\begin{array}{l}\text { El problema se identifica adecuadamente, pero presenta en su desarrollo dos } \\
\text { desviaciones aisladas y no justificadas del tema específico, y/o presenta información } \\
\text { reiterativa que afecta la comprensión fluida. }\end{array}$ \\
\hline & 1 & $\begin{array}{l}\text { El problema se identifica adecuadamente, sin embargo, en su desarrollo presenta datos } \\
\text { irrelevantes para su resolución y/o, a veces, contradictorios. }\end{array}$ \\
\hline & 0 & $\begin{array}{l}\text { El problema no se enuncia en el texto; y/o es imposible de comprender por la falta de } \\
\text { información o exceso de ella. }\end{array}$ \\
\hline \multirow[t]{5}{*}{$\begin{array}{l}\text { II. } \\
\text { Planteamiento } \\
\text { de tesis }\end{array}$} & 4 & $\begin{array}{l}\text { Se presenta la opinión del que escribe y desde dónde la realiza (profesor, alumno, } \\
\text { administrativo, sociedad, etc.) de manera explícita y precisa, utilizando marcas discursivas } \\
\text { adecuadas para destacarla. }\end{array}$ \\
\hline & 3 & $\begin{array}{l}\text { Se presenta la opinión del que escribe y desde dónde la realiza de manera explícita, } \\
\text { pero no se utilizan suficientes marcas discursivas para destacarla, lo que dificulta su } \\
\text { identificación inmediata. }\end{array}$ \\
\hline & 2 & $\begin{array}{l}\text { Se presenta un punto de vista impreciso que, si bien puede estar contextualizado, resulta } \\
\text { indeciso ante la problemática planteada. } \\
\text { O, se presenta explícitamente la opinión del que escribe, sin embargo, ocasionalmente } \\
\text { hay ambigüedad o no se especifica desde dónde se realiza esta opinión. }\end{array}$ \\
\hline & 1 & $\begin{array}{l}\text { Se presenta sistemáticamente un punto de vista impreciso y/o a veces contradictorio } \\
\text { respecto de la problemática planteada, dificultando la comprensión del texto. } \\
\text { O, se presenta explícitamente la opinión del que escribe, pero sistemáticamente hay } \\
\text { ambigüedad o no se especifica desde dónde se realiza esta opinión. }\end{array}$ \\
\hline & 0 & $\begin{array}{l}\text { No expresa una opinión respecto del problema planteado y/o no es posible identificarla } \\
\text { por ausencia de pistas textuales. }\end{array}$ \\
\hline \multirow[t]{5}{*}{$\begin{array}{l}\text { III. Relación } \\
\text { argumentativa }\end{array}$} & 4 & $\begin{array}{l}\text { Se articula la tesis con los argumentos de manera consistente y sistemática, se presentan } \\
\text { los argumentos con claridad y se explicita una relación coherente entre los argumentos. }\end{array}$ \\
\hline & 3 & $\begin{array}{l}\text { Se articula la tesis con los argumentos de manera consistente y sistemática, pero un } \\
\text { argumento se presenta con dificultad en su desarrollo y/o en su relación con } \\
\text { el resto de ellos. }\end{array}$ \\
\hline & 2 & $\begin{array}{l}\text { Se articula la tesis con los argumentos de manera consistente y sistemática, pero dos } \\
\text { argumentos se presentan con dificultad en su desarrollo y/o en su relación con } \\
\text { el resto de ellos. }\end{array}$ \\
\hline & 1 & $\begin{array}{l}\text { Se presenta la tesis con los argumentos de manera desarticulada, afectando su } \\
\text { identificación; y/o se presentan todos los argumentos como un listado de ideas. }\end{array}$ \\
\hline & 0 & $\begin{array}{l}\text { No hay tesis o se evidencia una ausencia de articulación entre la tesis y los argumentos; } \\
\text { y/o no existe una relación coherente entre los argumentos. }\end{array}$ \\
\hline
\end{tabular}




\begin{tabular}{|c|c|c|}
\hline \multirow[t]{5}{*}{$\begin{array}{l}\text { IV. } \\
\text { Identificación } \\
\text { de objeciones }\end{array}$} & 4 & $\begin{array}{l}\text { Se presentan objeciones posibles y posiciones rivales, y sistemáticamente se proveen } \\
\text { respuestas convincentes a estas, fortaleciendo los argumentos, ya sea ampliándolos, } \\
\text { restringiéndolos o especificándolos. }\end{array}$ \\
\hline & 3 & $\begin{array}{l}\text { Se presentan objeciones posibles y posiciones rivales, pero ocasionalmente se proveen } \\
\text { respuestas convincentes a estas, fortaleciendo los argumentos, ya sea ampliándolos, } \\
\text { restringiéndolos o especificándolos. }\end{array}$ \\
\hline & 2 & $\begin{array}{l}\text { Se presentan objeciones posibles y posiciones rivales, pero no se proveen respuestas } \\
\text { convincentes a estas que fortalezcan los argumentos. }\end{array}$ \\
\hline & 1 & $\begin{array}{l}\text { Se presentan ocasionalmente objeciones posibles y posiciones rivales, pero estas nunca } \\
\text { son respondidas. }\end{array}$ \\
\hline & 0 & No se presentan objeciones y posiciones rivales. \\
\hline \multirow[t]{5}{*}{ V. Valoración } & 4 & $\begin{array}{l}\text { Se enuncian sistemáticamente juicios de valor atingentes al desarrollo del problema y su } \\
\text { resolución a través de marcas de valoración. }\end{array}$ \\
\hline & 3 & $\begin{array}{l}\text { Se presentan sistemáticamente juicios de valor del problema y del desarrollo de la } \\
\text { resolución, pero en una oportunidad las interpretaciones resultan ambiguas, y/o no se } \\
\text { justifican cuando hay necesidad, y/o no son atingentes. }\end{array}$ \\
\hline & 2 & $\begin{array}{l}\text { Se presentan juicios de valor del problema y su resolución, pero en dos oportunidades } \\
\text { las interpretaciones resultan ambiguas, y/o no se justifican cuando hay necesidad, y/o } \\
\text { no son atingentes. }\end{array}$ \\
\hline & 1 & $\begin{array}{l}\text { Se presentan juicios de valor del problema y su resolución, pero sistemáticamente estas } \\
\text { resultan ambiguas, contradictorias, incoherentes, no justificadas o no atingentes. }\end{array}$ \\
\hline & 0 & $\begin{array}{l}\text { No se realizan juicios de valor de los elementos fundamentales que forman parte de los } \\
\text { problemas, situaciones o dilemas planteados. }\end{array}$ \\
\hline \multirow[t]{5}{*}{$\begin{array}{l}\text { VI. Inferencia } \\
\text { de resolución }\end{array}$} & 4 & $\begin{array}{l}\text { Se presentan una o más soluciones viables a la problemática presentada de manera } \\
\text { crítica y concisa, derivándose del desarrollo argumentativo y con sugerencias atingentes. }\end{array}$ \\
\hline & 3 & $\begin{array}{l}\text { Se presentan una o más soluciones viables de manera crítica y concisa, derivándose del } \\
\text { desarrollo argumentativo y con sugerencias atingentes, pero la falta de síntesis dificulta } \\
\text { su comprensión. }\end{array}$ \\
\hline & 2 & $\begin{array}{l}\text { Se presentan una o más soluciones viables a los posibles problemas, que aunque claras y } \\
\text { realistas, son superficiales. Además, las sugerencias a veces son imprecisas. }\end{array}$ \\
\hline & 1 & $\begin{array}{l}\text { La o las soluciones que se presenta(n) resulta(n) insuficiente(s) o incoherente(s) con el } \\
\text { conflicto planteado. }\end{array}$ \\
\hline & 0 & No presenta solución alguna a los posibles problemas, situaciones o dilemas presentados. \\
\hline \multirow[t]{5}{*}{$\begin{array}{l}\text { VII. Análisis } \\
\text { sistémico }\end{array}$} & 4 & $\begin{array}{l}\text { Se integran sistemáticamente varias perspectivas o actores de la problemática planteada, } \\
\text { más allá del punto de vista individual, mostrándose una visión panorámica del problema. }\end{array}$ \\
\hline & 3 & $\begin{array}{l}\text { Se integran sistemáticamente varias perspectivas o actores de la problemática planteada, } \\
\text { más allá del punto de vista individual, pero esta integración resulta incompleta o está } \\
\text { parcialmente desarrollada. }\end{array}$ \\
\hline & 2 & $\begin{array}{l}\text { Se integran sistemáticamente varias perspectivas o actores de la problemática planteada, } \\
\text { sin embargo, no se realiza esto de manera explícita y requiere una inferencia de parte } \\
\text { del lector. }\end{array}$ \\
\hline & 1 & Se presenta una sistematización basada únicamente en el punto de vista individual. \\
\hline & 0 & No se presenta una integración, pues los puntos de vista no están definidos. \\
\hline
\end{tabular}

Fuente: elaboración propia

La tabla anterior expone el Instrumento de evaluación de habilidades escritas de pensamiento crítico y la descripción de cada dimensión, según rango de puntaje, lo que es, efectivamente, una propuesta que se sustenta en la perspectiva teórica actual, respecto a la reflexión pedagógica, el pensamiento crítico y su importancia en la pedagogía contemporánea. 
b. Describir el desempeño de los estudiantes que recién están comenzando su formación docente en las habilidades de pensamiento crítico, de manera que les permitirán desarrollar una futura reflexión pedagógica.

Para el cumplimiento de este objetivo, se definió el mismo parámetro aplicado por la institución en la que están matriculados los participantes del estudio para determinar la aprobación en las evaluaciones académicas, es decir, el 70\% de exigencia. En estos términos, y considerando en primera instancia los puntajes totales obtenidos por texto, a continuación, se grafica el porcentaje de logro total de los estudiantes en el Instrumento de evaluación de habilidades escritas de pensamiento crítico.

\section{Gráfico 1}

Porcentaje de logro en el Instrumento de evaluación de habilidades escritas de pensamiento crítico

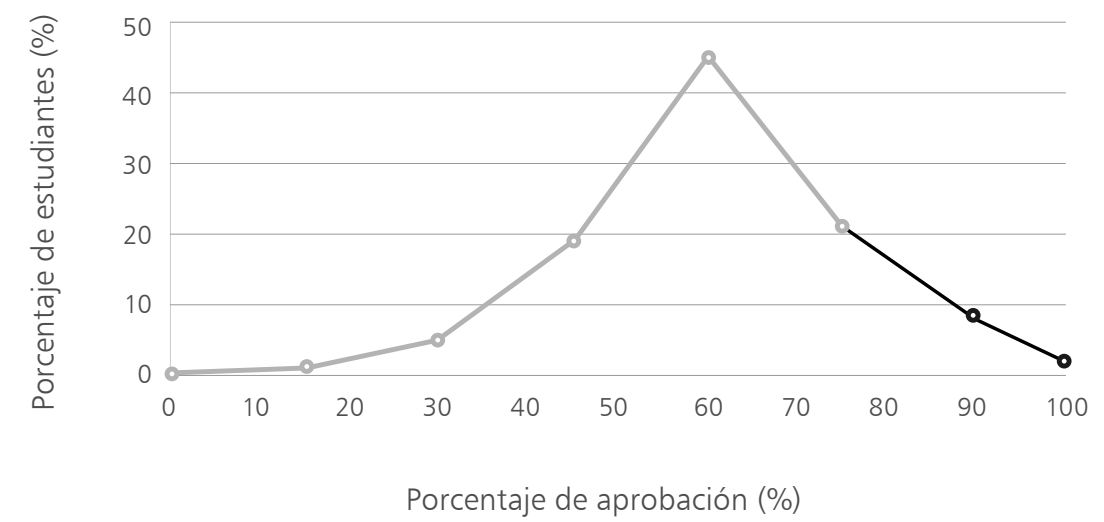

Fuente: elaboración propia

De los 265 textos que componen la muestra, es importante destacar que ninguno (0\%) logra lo que se espera, en términos del desarrollo textual, sobre habilidades de pensamiento crítico y, además, solo el $22.26 \%$ de los textos ( $n=59$; tramo representado en la línea negra) tiene porcentajes de logro iguales o mayores al $70 \%$ de exigencia. Si en términos de puntaje total (28, dados 4 puntos totales por dimensión), se entiende que lograr el $70 \%$ equivale a obtener 20 puntos o más; el hecho de que la media sea de 15.72, y la desviación estándar de 4.25, indica que la mayoría de los textos pueden ser calificados como no logrados, pues una gran cantidad fluctúa entre los 11.47 y 19.97 puntos, sin alcanzar ese $70 \%$ de exigencia.

Es interesante, además, analizar el rendimiento de los estudiantes a partir de las dimensiones de cada rúbrica: 
Tabla 3

Análisis de resultados por dimensión del Instrumento de evaluación

de habilidades escritas de pensamiento crítico

\begin{tabular}{l|c|c|c|c|c|c}
\hline \multicolumn{1}{l|}{ Dimensión } & $\mathbf{n} \geq \mathbf{7 0} \%$ & $\mathbf{\%}$ & $\mathbf{n} \mathbf{7 0} \%$ & $\mathbf{\%}$ & $\begin{array}{c}\text { Media } \\
\text { Desviación } \\
\text { estándar }\end{array}$ \\
\hline I. Identificación del problema según contexto & 144 & 54.3 & 121 & 45.6 & 2.60 & 1.07 \\
\hline II. Planteamiento de tesis & 126 & 47.7 & 139 & 52.4 & 2.40 & 0.97 \\
\hline III. Relación argumentativa & 90 & 33.9 & 175 & 66 & 2.09 & 1.03 \\
\hline IV. Identificación de objeciones & 80 & 30.1 & 185 & 69.8 & 1.89 & 1.09 \\
\hline V. Valoración & 118 & 44.5 & 147 & 55.4 & 2.40 & 1.05 \\
\hline VI. Inferencia de resolución & 79 & 29.8 & 186 & 70.1 & 1.87 & 1.15 \\
\hline VII. Análisis sistémico & 128 & 48.3 & 137 & 51.6 & 2.44 & 1.04 \\
\hline
\end{tabular}

Fuente: elaboración propia

Como se observa, no existe una diferencia significativa entre los resultados obtenidos en cada dimensión, respecto a la frecuencia o porcentaje de textos que alcanza el $70 \%$ del logro. En otras palabras, en la mayoría de las dimensiones hubo alrededor de un $41.22 \%$ de textos que lograron esta exigencia, pudiendo obtener 3 o 4 puntos en cada dimensión. Como extremos, es posible destacar la dimensión I (Identificación del problema según contexto) como la que presenta un mayor porcentaje de logro (54.3\% de los textos alcanzan la exigencia del $70 \%$ en este aspecto), y la dimensión VI (Inferencia de resolución) como uno de los puntos en el que menos textos cumplen con este $70 \%$ de logro.

En cuanto a la revisión realizada por el Instrumento de evaluación de aspectos formales y textuales (cuyo puntaje total es de 15 puntos, siendo 3 el máximo posible por dimensión), en general los textos presentan mejores resultados que en el Instrumento de evaluación de habilidades escritas de pensamiento crítico, considerando que el $41.88 \%$ de la muestra alcanza el $70 \%$ de exigencia (equivalente a 11 puntos o más), y dos textos el 100\% de logro.

Tabla 4

Análisis de resultados por dimensión del Instrumento de aspectos formales y textuales

\begin{tabular}{l|c|c}
\hline Dimensión & Media & $\begin{array}{c}\text { Desviación } \\
\text { estándar }\end{array}$ \\
\hline 1. Coherencia oracional & 2.13 & 0.82 \\
\hline 2. Coherencia interpárrafos & 2.31 & 0.69 \\
\hline 3. Uso de conectores & 2.25 & 0.74 \\
\hline 4. Convenciones ortográficas & 1.30 & 0.78 \\
\hline 5. Variedad y adecuación léxica & 2.11 & 0.74
\end{tabular}

Fuente: elaboración propia. 
Al comparar cada dimensión el rendimiento también es similar, a excepción de la dimensión 4 (Ortografía literal, puntual y acentual), en la cual el puntaje tiende a ser mucho más bajo (media: 1.30 de 3 puntos posibles).

c. Determinar la incidencia de cada habilidad o dimensión del instrumento en un producto escrito que evidencie un alto nivel de pensamiento crítico.

Con el fin de lograr este objetivo, se presenta el siguiente estudio correlacional, aplicando el coeficiente de Spearman debido a que las variables no se distribuyen normalmente (prueba K-S):

Tabla 5

Estudio correlacional entre las dimensiones y el puntaje total del Instrumento de evaluación de habilidades escritas de pensamiento crítico

\begin{tabular}{|c|c|c|}
\hline \multicolumn{2}{|l|}{ Correlaciones } & \multirow{2}{*}{$\begin{array}{c}\text { Puntaje Total } \\
0.484^{* *}\end{array}$} \\
\hline I. Identificación del problema según contexto & Coeficiente de correlación & \\
\hline & Sig. (bilateral) & 0.000 \\
\hline & $\mathrm{N}$ & 265 \\
\hline \multirow[t]{3}{*}{ II. Planteamiento de tesis } & Coeficiente de correlación & $0.546^{* *}$ \\
\hline & Sig. (bilateral) & 0.000 \\
\hline & $\mathrm{N}$ & 265 \\
\hline \multirow[t]{3}{*}{ III. Relación argumentativa } & Coeficiente de correlación & $0.654^{* *}$ \\
\hline & Sig. (bilateral) & 0.000 \\
\hline & $\mathrm{N}$ & 265 \\
\hline \multirow[t]{3}{*}{ IV. Identificación de objeciones } & Coeficiente de correlación & $0.357^{* *}$ \\
\hline & Sig. (bilateral) & 0.000 \\
\hline & $\mathrm{N}$ & 265 \\
\hline \multirow[t]{3}{*}{ V. Valoración } & Coeficiente de correlación & $0.599^{* *}$ \\
\hline & Sig. (bilateral) & 0.000 \\
\hline & $\mathrm{N}$ & 265 \\
\hline \multirow[t]{3}{*}{ VI. Inferencia de resolución } & Coeficiente de correlación & $0.583^{* *}$ \\
\hline & Sig. (bilateral) & 0.000 \\
\hline & $\mathrm{N}$ & 265 \\
\hline \multirow[t]{3}{*}{ VII. Análisis sistémico } & Coeficiente de correlación & $0.635^{* *}$ \\
\hline & Sig. (bilateral) & 0.000 \\
\hline & $\mathrm{N}$ & 265 \\
\hline
\end{tabular}

** La correlación es significativa en el nivel 0.01 (bilateral).

Fuente: elaboración propia.

Como se observa, existe una buena correlación (entre 0.6 y 0.8) entre las dimensiones III (Relación argumentativa) y VII (Análisis sistémico) y el puntaje total, siendo estas las dimensiones que mejor pueden predecir la elaboración de un texto escrito que evidencie un alto nivel de pensamiento crítico. El resto de las dimensiones presenta una correlación moderada con el puntaje total. 
d. Estimar las relaciones entre las dimensiones textuales (coherencia, adecuación léxica y ortografía) y un producto escrito que evidencie un alto nivel de pensamiento crítico.

Finalmente, se exponen las correlaciones entre las dimensiones de ambas rúbricas, con el objetivo de establecer y determinar la vinculación entre las habilidades y procesos más cognitivos, asociados al pensamiento crítico y la argumentación (Instrumento de evaluación de habilidades escritas de pensamiento crítico, 11) y aquellos tradicionalmente circunscritos en los parámetros de discurso escrito (Instrumento de evaluación de aspectos formales y textuales, 12):

Tabla 6

Estudio correlacional entre el Instrumento de evaluación de habilidades escritas de pensamiento crítico y el Instrumento de evaluación de aspectos formales y textuales

\begin{tabular}{|c|c|c|c|c|c|c|c|}
\hline Dimensiones I1 & Dimensiones 12 & 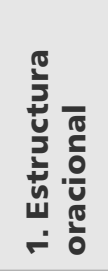 & 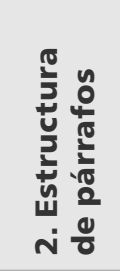 & 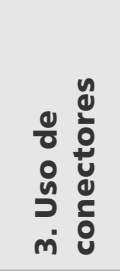 & 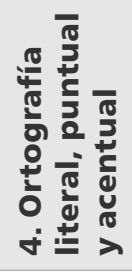 & 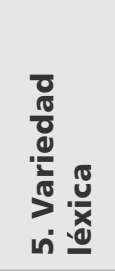 & \begin{tabular}{l}
$\overline{0}$ \\
$\stackrel{0}{0}$ \\
\multirow{2}{0}{} \\
$\frac{0}{2}$ \\
$\stackrel{0}{5}$ \\
$\frac{5}{2}$
\end{tabular} \\
\hline \multirow{3}{*}{$\begin{array}{l}\text { I. Identificación } \\
\text { del problema } \\
\text { según contexto }\end{array}$} & Coeficiente de correlación & 0.001 & $0.187^{* *}$ & $0.172^{* *}$ & 0.053 & $0.140^{*}$ & \\
\hline & Sig. (bilateral) & 0.985 & 0.003 & 0.006 & 0.400 & 0.025 & \\
\hline & $\mathrm{N}$ & 259 & 259 & 259 & 257 & 258 & \\
\hline \multirow{3}{*}{$\begin{array}{l}\text { II. Planteamiento } \\
\text { de tesis }\end{array}$} & Coeficiente de correlación & 0.115 & 0.121 & $0.197^{* *}$ & -0.041 & $0.150^{*}$ & \\
\hline & Sig. (bilateral) & 0.064 & 0.052 & 0.001 & 0.512 & 0.016 & \\
\hline & $\mathrm{N}$ & 259 & 259 & 259 & 257 & 258 & \\
\hline \multirow{3}{*}{$\begin{array}{l}\text { III. Relación } \\
\text { argumentativa }\end{array}$} & Coeficiente de correlación & 0.081 & $0.178^{* *}$ & $0.149^{*}$ & 0.017 & 0.104 & \\
\hline & Sig. (bilateral) & 0.195 & 0.004 & 0.016 & 0.785 & 0.095 & \\
\hline & $\mathrm{N}$ & 259 & 259 & 259 & 257 & 258 & \\
\hline \multirow{3}{*}{$\begin{array}{l}\text { IV. Identificación } \\
\text { de objeciones }\end{array}$} & Coeficiente de correlación & 0.089 & 0.081 & 0.043 & -0.029 & -0.005 & \\
\hline & Sig. (bilateral) & 0.153 & 0.193 & 0.488 & 0.638 & 0.930 & \\
\hline & $\mathrm{N}$ & 259 & 259 & 259 & 257 & 258 & \\
\hline \multirow[t]{3}{*}{ V. Valoración } & Coeficiente de correlación & $0.157^{*}$ & $0.278^{* *}$ & $0.162^{* *}$ & $0.138^{*}$ & $0.180^{* *}$ & \\
\hline & Sig. (bilateral) & 0.011 & 0.000 & 0.009 & 0.027 & 0.004 & \\
\hline & $\mathrm{N}$ & 259 & 259 & 259 & 257 & 258 & \\
\hline \multirow{3}{*}{$\begin{array}{l}\text { VI. Inferencia } \\
\text { de resolución }\end{array}$} & Coeficiente de correlación & 0.104 & $0.176^{* *}$ & 0.118 & -0.077 & -0.032 & \\
\hline & Sig. (bilateral) & 0.094 & 0.004 & 0.058 & 0.220 & 0.605 & \\
\hline & $\mathrm{N}$ & 259 & 259 & 259 & 257 & 258 & \\
\hline \multirow{3}{*}{$\begin{array}{l}\text { VII. Análisis } \\
\text { sistémico }\end{array}$} & Coeficiente de correlación & 0.115 & $0.318^{* *}$ & $0.225^{* *}$ & -0.021 & $0.129^{*}$ & \\
\hline & Sig. (bilateral) & 0.065 & 0.000 & 0.000 & 0.743 & 0.039 & \\
\hline & $\mathrm{N}$ & 259 & 259 & 259 & 257 & 258 & \\
\hline \multirow[t]{3}{*}{ Puntaje total } & Coeficiente de correlación & & & & & & $0.262 * *$ \\
\hline & Sig. (bilateral) & & & & & & 0.000 \\
\hline & $\mathrm{N}$ & & & & & & 257 \\
\hline
\end{tabular}

** La correlación es significativa en el nivel 0.01 (bilateral).

Fuente: elaboración propia. 
En general, las correlaciones entre las dimensiones de ambas rúbricas son mínimas, lo que prácticamente indica que el rendimiento en cualquier dimensión de la primera no afecta al rendimiento de los aspectos formales de escritura evaluados en la segunda, y viceversa. En términos de puntaje global, la correlación es baja entre los dos instrumentos, por lo que es posible afirmar que, estadísticamente, el desempeño en una rúbrica no puede predecir los resultados de la otra.

\section{Discusión}

Los resultados obtenidos en este estudio en relación con el propósito de investigación reportan un procedimiento para evaluar habilidades de pensamiento crítico que fundamentan la reflexión pedagógica, desde un instrumento aplicable a un texto escrito predominantemente argumentativo. De esta manera, se construyó una herramienta de evaluación sustentada en una línea teórica contemporánea que engloba las concepciones actuales de reflexión pedagógica, pensamiento crítico, argumentación razonada y discurso académico a partir de siete dimensiones. Estas conforman a su vez un parámetro global de lo que sería un texto en el que se desarrollan las habilidades de pensamiento crítico involucradas en procesos de reflexión pedagógica.

Respecto de este parámetro general, se observa un bajo rendimiento global que expone las dificultades que tienen los estudiantes al poner en práctica sus habilidades de pensamiento crítico en la producción escrita, lo que es consistente con investigaciones previas (Carlino, 2004; Errázuriz-Cruz et al., 2015; Navarro, 2014; Salinas et al., 2018).

Específicamente, uno de los aspectos que se destaca al analizar los porcentajes de logro en cada dimensión evaluada en el Instrumento de evaluación de habilidades escritas de pensamiento crítico es que, al no existir diferencias significativas en el desempeño de estas, todas las dimensiones representan un impedimento para el logro de dicha tarea. Cada una de ellas es un aspecto en que los y las estudiantes que ingresan a la educación superior demuestran una baja competencia. De esta manera, contrariamente a lo esperado respecto a que ciertas habilidades y procesos pudieran resultar más complejos que otros, como por ejemplo la incorporación de voces (IV) y el análisis sistémico (VII), las dificultades no se centralizan en algunos procesos, sino que, efectivamente, se genera un alto porcentaje de textos escritos desintegrados, carentes de un hilo conductor o de fuerza argumentativa, incumpliéndose la esencia del pensamiento crítico (Córdova et al., 2016; García y Barrientos, 2009; Larrivee, 2000). 
Además de las dimensiones que obtuvieron un mejor y peor desempeño, esto es I (Identificación del problema según contexto) y VI (Inferencia de resolución), respectivamente, al realizar el estudio correlacional resalta, además, las dimensiones III (Relación argumentativa) y VII (Análisis sistémico) como aquellas que mejor pueden predecir la elaboración de un texto escrito que evidencie un alto nivel de pensamiento crítico. Dado que ambas dimensiones tienen en común descriptores relacionados con el andamiaje e integración textual sistemática que da cuenta de la línea argumentativa, es posible afirmar que el instrumento privilegia esta concepción analítica de pensamiento crítico que se pone en práctica en cualquier tarea de reflexión pedagógica (Scriven \& Fisher, 1997; American Psycological Association, 1990).

Por otro lado, al correlacionar ambas rúbricas, los datos demuestran una mínima incidencia entre las habilidades de pensamiento crítico y los aspectos formales y textuales de escritura, lo que para una didáctica de la escritura indicaría que desarrollar, por sí solos, contenidos tales como ortografía, coherencia intraoracional e interpárrafos no contribuye a desarrollar el pensamiento crítico y viceversa. En términos de formar pensamiento crítico, más que la preocupación por los estándares formales, actualmente tiene más sentido la integración de la lectura y la escritura desde su función epistémica, es decir, crear conocimiento mediante la escritura, crear conocimiento a partir de la comprensión lectora de diferentes voces o fuentes (Solé et al., 2005).

\section{Conclusiones}

Tras el análisis de los resultados obtenidos con respecto a los objetivos planteados en el estudio, se concluye que:

1. El Instrumento de evaluación de habilidades escritas de pensamiento crítico permite medir siete dimensiones definitorias de la reflexión pedagógica mediante una actividad de escritura.

2. Al aplicar este instrumento en textos producidos por estudiantes recién ingresados a diferentes carreras de pedagogía, los resultados indican que estos presentan bajos niveles de desarrollo de tales dimensiones.

3. Las competencias para incorporar diferentes puntos de vista, insertar marcas de juicios de valoración coherentes con el punto de vista propio y articular este con argumentos coherentes entre sí, son algunas de las que más pueden predecir la elaboración de un texto escrito de alta calidad argumentativa, lo que para las implicancias 
pedagógicas puede significar que reforzar estas habilidades desde la escritura en los programas de alfabetización académica podría ser una posibilidad para generar una mejora en el desarrollo de las capacidades de reflexión pedagógica en los estudiantes de formación docente.

4. Más allá de la importancia formal que se le ha dado al uso de reglas ortográficas, la coherencia y cohesión en la relación de párrafos y oraciones, y a la utilización de un léxico preciso, en el contexto de desarrollo de habilidades de pensamiento crítico y reflexión pedagógica estas capacidades parecen no tener incidencia (al menos estadística, pues no es posible afirmar que no hay una relación en términos de procesos de organización textual y discursiva) en la elaboración de un texto argumentativo que refleje competencias críticas.

En definitiva, la investigación expuesta es una evidencia para continuar instalando el pensamiento crítico y todas las habilidades que implica, como un aspecto transversal y obligatorio en las asignaturas de los programas de formación de profesores y profesoras, a través de una cautelosa planificación y una permanente evaluación que demuestre el progreso de los estudiantes en estos procesos que fundamentan la construcción de reflexión pedagógica. Como se observa en el estudio, esta no es una competencia que se aprenda de manera natural ni está interiorizada en los estudiantes que egresan de los colegios, sino que supone la interiorización de una lógica de aproximación a los contextos educativos a través de una organización retórico-discursiva que revele un exhaustivo análisis relacional entre los actores y factores que intervienen en estos. En esta línea, la aplicación de instancias formativas y evaluativas que exijan la construcción de textos argumentativos resulta esencial en los programas de alfabetización académica de la formación docente.

Es importante considerar que estos resultados se radican en una universidad de la Región Metropolitana, y que, si bien no representan en su totalidad a los estudiantes que ingresan a las diversas carreras de pedagogía en Chile, pueden orientar una idea respecto al estado de las competencias de pensamiento crítico en los alumnos y alumnas recién egresados del sistema escolar. Evidentemente, en futuras investigaciones, debería indagarse si estos datos son específicos de la universidad estudiada o si, por el contrario, se repiten en otras instituciones de educación superior, y asimismo, podría resultar interesante explorar si existen variables socioculturales de los sujetos analizados que expliquen los resultados. Paralelamente, otro aspecto que podría desarrollarse más en futuras investigaciones es la 
profundización de cada dimensión del pensamiento crítico explorada en este estudio, y la valoración que profesores y estudiantes otorgan a estas respecto a lo que consideran un texto de alta calidad en términos del pensamiento crítico. De tal manera, se puede continuar la sistematización de los procesos y las habilidades que los futuros profesores deben adquirir para el desarrollo de la reflexión pedagógica.

En este sentido más bien constructivo y resolutivo, más allá de entender por qué los resultados son bajos al exigir una tarea escrita que refleje una lógica argumentativa crítica, queda pendiente por investigar los criterios, métodos e instrumentos evaluativos que, a juicio de los formadores, jefes de carreras y sus estudiantes, contribuyen a desarrollar la reflexión pedagógica escrita, siendo necesario profundizar aún más en la interacción entre las prácticas de escritura y el desarrollo del pensamiento crítico en la formación docente.

En el contexto del proyecto mayor, el Instrumento de evaluación de habilidades escritas de pensamiento crítico constituye un aporte para evaluar progresivamente las habilidades y los procesos involucrados en el desarrollo de reflexión pedagógica en diversos géneros discursivos escritos y contextos académicos, permitiendo aproximarse al desempeño de los futuros profesores, como por ejemplo, en la Prueba de Conocimientos Pedagógicos Generales de la END-FID. Conjuntamente, se espera que la aplicación sistemática de la evaluación del pensamiento crítico mediante actividades de escritura permita que los estudiantes vayan adquiriendo los aprendizajes necesarios para lograr una reflexión pedagógica que se ajuste a los requerimientos de la labor docente, y que en cada etapa los alumnos y alumnas vayan construyendo mejores textos en términos de lo que esta competencia exige.

\section{Sobre los autores}

Nicole Schnitzler-Sommerfeld es lingüista. Máster en Trastornos de la Comunicación y del Lenguaje.

Paulina Núñez-Lagos es académica investigadora. Línea de investigación: sicolingüística de la educación.

\section{Referencias bibliográficas}

American Psycological Association (APA) (1990). Pensamiento Crítico: Una Declaración De Consenso De Expertos Con Fines De Evaluación E Instrucción Educativa. "El Informe Delphi", Comité Preuniversitario de Filosofía. The California Academia Press, Millbrae, Canada. 
Bajtín, M. (1981). The Dialogic Imagination (C. Emerson \& M. Holquist, trads.). University of Texas Press.

Beauchamp, C. (2015). Reflection in teacher education: issues emerging from a review of current literature. Reflective Practice, 16(1), 123-141. https://doi. org/10.1080/14623943.2014.982525

Camps, A. \& Castelló, M. (2013). La escritura académica en la universidad. REDU - Revista de Docencia Universitaria. Número Monográfico dedicado a Academic Writing, 11(1), 17-36. http://www.red-u.net

Carlino, P. (2004). El proceso de escritura académica: cuatro dificultades de la enseñanza universitaria. Educere, Revista Venezolana de Educación, 8(26), 321-327. https://www.redalyc.org/pdf/356/35602605.pdf

Carreras de pedagogía - CPEIP. (2019). https://www.cpeip.cl/formacion-inicialdocente/

Collin, S., Karsenti, T. \& Komis, V. (2013). Reflective practice in initial teacher training: Critiques and perspectives. Reflective practice, 14(1), 104-117. https://doi.org/10.1080/14623943.2012.732935

Concha, S., Hernández, C., Del Río, F., Romo, F. \& Andrade, L. (2013). Reflexión pedagógica en base a casos y dominio de lenguaje académico en estudiantes de cuarto año de Pedagogía en Educación Básica. Calidad en la educación, (38), 81-113. http://dx.doi.org/10.4067/S0718-45652013000100002

Córdova, A., Velásquez, M. \& Arenas, L. (2016). El rol de la argumentación en el pensamiento crítico y en la escritura epistémica en Biología e Historia: aproximación a partir de las representaciones sociales de los docentes. Alpha (Osorno), (43), 39-55. https://www.scielo.cl/pdf/alpha/n43/art_04.pdf

Darling-Hammond, L. \& Bransford, J. (Eds.) (2005). Preparing teachers for a changing world. What teachers should learn and be able to do. Jossey-Bass.

Errázuriz Cruz, M. C., Arriagada Rubilar, L., Contreras Villablanca, M. \& López Westermeier, C. (2015). Diagnóstico de la escritura de un ensayo de alumnos novatos de Pedagogía en el campus Villarica UC, Chile. Perfiles educativos, 37(150), 76-90. http://www.scielo.org.mx/scielo.php?script=sci_artt ext\&pid $=$ S0185-26982015000400005

Freire, P. (1997). A la sombra de este árbol. Editorial Roure.

Fund, Z., Court, D. \& Kramarski, C. (2002). Construction and application of an evaluative tool to assess reflection in teacher training courses. Assessment and Evaluation in Higher Education, 27(6), 485-499. https://doi.org/10.108 0/0260293022000020264

García, A. \& Barrientos, O. (2009) Los dominios del pensamiento crítico: una lectura desde la teoría de la educación. Teoría de la Educación. Revista Interuniversitaria, 21(2), 19-44. https://doi.org/10.14201/7150

Hatton, N., \& Smith, D. (1995). Reflection in teacher education: Towards definition and implementation. Teaching and teacher education, 11(1), 33-49. https://www.sciencedirect.com/science/article/abs/pii/0742051X9400012U

Jarpa, M. \& Becerra, N. (2019). Escritura para la reflexión pedagógica: concepciones y géneros discursivos que escriben los estudiantes en dos carreras de pedagogía. Logos (La Serena), 29(2), 364-381. http://dx.doi.org/10.15443/rl2928

Lamas, P. \& Vargas-D'Uniam, J. (2016). Los niveles de reflexión en los portafolios de la Práctica Pre Profesional Docente. REDU. Revista de Docencia Universitaria, 14(2), 57-78. http://dx.doi.org/10.4995/redu.2016.5680 
Lara - Subiabre, B. (2019). Reflexión pedagógica de profesores en formación. Un estudio de cuatro universidades chilenas. Perspectiva Educacional, 58(1), 4-25. http://dx.doi.org/10.4151/07189729-vol.58-iss.1-art.802

Larrivee, B. (2000). Transforming Teaching Practice: becoming the critically reflective teacher. Reflective Practice, 1(3), 293-307. https://doi.org/10.10 80/713693162

Larrivee B. (2008). Development of a tool to assess teachers' level of reflective practice. Reflective Practice, 9(3), 341-360. https://doi.org/10.1080/1462394 0802207451

Luk, J. (2008). Assessing teacher practicum reflections: Distinguishing discourse features of the "high" and "low" grade reports. System, (36), 624-641. https://doi.org/10.1016/j.system.2008.04.001

Martin, J. R. \& White, P. R. (2003). The language of evaluation (Vol. 2). Palgrave Macmillan.

MINEDUC. (2019). Evaluación Nacional Diagnóstica de la Formación Inicial Docente. https://www.diagnosticafid.cl/

Moon, J. (2007). Getting the measure of reflection: considering matters of definition and depth. Journal of Radiotherapy in Practice, 6(4), 191-200. https:// www.cambridge.org/core/journals/journal-of-radiotherapy-in-practice/arti cle/getting-the-measure-of-reflection-considering-matters-of-definitionand-depth/DFF281CADA91D3502D26D43CDD217BA7

Navarro, F. (coord.) (2014). Géneros discursivos e ingreso a las culturas disciplinares. Aportes para una didáctica de la lectura y la escritura en educación superior. En Manual de escritura para carreras de humanidades (pp. 29-52). Universidad de Buenos Aires (UBA).

Núñez, P. \& Espejo, C. (2005). Estudio exploratorio acerca de la conceptualización del informe escrito en el ámbito académico. En A. M. Harvey (Ed.), En torno al discurso. Contribuciones de América Latina (pp. 135-148). Ediciones Pontificia Universidad Católica.

Ossa-Cornejo, C. J., Palma-Luengo, M. R., Martín, L. S., Nelly, G., Quintana-AbeIlo, I. M. \& Díaz-Larenas, C. H. (2017). Análisis de instrumentos de medición del pensamiento crítico. Ciencias Psicológicas, 11(1), 19-28. https://www. redalyc.org/journal/4595/459551482003/html/

Parodi, G. (Ed.) (2010). Alfabetización académica y profesional en el siglo XXI: leer y escribir desde las disciplinas. Planeta.

Pérez Gómez, A. (2007). Cuadernos de educación 1. La naturaleza de las competencias básicas y sus aplicaciones pedagógicas. http://www.slideshare.net/ laloreyes23/naturaleza-de-las-competencias-basicas

Rodrigues, R. (2013). El desarrollo de la práctica reflexiva sobre el quehacer docente, apoyada en el uso de un portafolio digital, en el marco de un programa de formación para académicos de la Universidad Centroamericana de Nicaragua [Tesis de Doctorado]. http://hdl.handle.net/2445/43124

Salinas, Á., Rozas, T. \& Cisternas, P. (2018). El foco y la profundidad de la reflexión docente en estudiantes de pedagogía en Chile. Perfiles educativos, 40(161), 87-106. http://www.scielo.org.mx/scielo.php?script=sci_arttext\& pid $=$ S0185-26982018000300087 
Schön, D. (1992). La formación de profesionales reflexivos. Hacia un nuevo diseño de la enseñanza y el aprendizaje en los profesionales. Paidós.

Schön, D. (1998). El profesional reflexivo: cómo piensan los profesionales cuando actúan. Paidós.

Scriven, M. \& Fisher, A. (1997) Critical thinking. Its definition and assessment. Centre for Research in Critical Thinking: Edgepress.

Silvestri, A. (2001). La producción de la argumentación razonada en el adolescente: Las falacias de aprendizaje. En M. C. Martínez (Ed.), Aprendizaje de la argumentación razonada. Desarrollo temático de los textos expositivos y argumentativos (vol. 3, pp. 29-48). Cátedra UNESCO para la Lectura y la Escritura, Universidad del Valle.

Snow, C. \& Uccelli, P. (2009). The challenge of academic language. En D. Olson y N. Torrance (Eds.), The Cambridge Handbook of Literacy (pp. 112-133). Cambridge University Press.

Solé, I., Mateos, M., Miras, M., Martín, E., Castells, N., Cuevas, I. \& Grácia, M. (2005). Lectura, escritura y adquisición de conocimientos en Educación Secundaria y Educación Universitaria. Infancia y Aprendizaje, 28(3), 329-347. https://doi.org/10.1174/0210370054740241

Sologuren, E. (2019). Approche de l'ecrit academique dans un milieu d'apprentissage de l'ingenierie civile en informatique. Policromias-Revista de Estudos do Discurso, Imagem e Som, 4(2), 51-65. https://revistas.ufrj.br/index.php/ policromias/article/view/27855/18007

Sparks-Langer, G. M., Simmons, J. M., Pasch, M., Colton, A. \& Starko, A. (1990). Reflective pedagogical thinking: how can we promote it and measure it? Journal of Teacher Education, (41), 23-32. https://doi.org/10.1177/0022 48719004100504

Tapia-Ladino, M. \& Burdiles Fernández, G. (2009). Una caracterización del género informe escrito. Letras, 51(78), 17-49. http://ve.scielo.org/scielo.php?script= sci_arttext\&pid=S0459-12832009000100001

Van Dijk, T. \& Kinstch, W. (1983). Strategies of discourse comprehension. Academy Press.

Van Manen, M. (1977). Linking ways of knowing with ways of being practical. Curriculum Inquiry, (6), 205-228. https://doi.org/10.2307/1179579

Venegas, R., Zamora, S. \& Galdames, A. (2016). Hacia un modelo retórico-discursivo del macrogénero Trabajo Final de Grado en Licenciatura. Signos, (49), 247-279. https://scielo.conicyt.cl/pdf/signos/v49s1/art12.pdf

Youngs, P. \& Bird, T. (2010). Using embedded assessments to promote pedagogical reasoning among secondary teaching candidates. Teacher and teaching education, 26(2), 185-198. https://www.infona.pl/resource/bwmeta1.ele ment.elsevier-e753c257-1f5f-38db-8f08-fc39abcd038e

Zeichner, K. M. (1987). Preparing reflective teachers: An overview of instructional strategies which have been employed in preservice teacher education. International Journal of Educational Research, 11(5), 565-575. https://doi. org/10.1016/0883-0355(87)90016-4 